Acta Botanica Mexicana (2002), 61: 27-33

\title{
DOS ESPECIES NUEVAS DE ORQUÍDEAS DEL ESTADO DE MORELOS, MÉXICO
}

\author{
Adolfo Espejo Serna \\ Ana Rosa López-FerRari \\ Herbario Metropolitano \\ Departamento de Biología \\ Apartado postal 55-535 \\ 09340 México, D.F. \\ email: aes@xanum.uam.mx \\ ROLANDO JiMÉNEZ MACHORRO \\ LUIS SÁnchez SALDAÑa \\ Y \\ Javier García Cruz \\ Herbario AMO \\ Apartado postal 53-123 \\ 11520 México, D.F. \\ email: eric@internet.com.mx
}

División de Ciencias Biológicas y de la Salud

Universidad Autónoma Metropolitana-Iztapalapa

\section{RESUMEN}

Se describen e ilustran dos nuevas especies del estado de Morelos. Malaxis ribana Espejo \& López-Ferrari y Habenaria uncata R. Jiménez, L. Sánchez \& García-Cruz. Ambas provienen de los alrededores de Cuernavaca.

Palabras clave: Habenaria, Malaxis, México, Morelos, Orchidaceae.

\section{ABSTRACT}

Two new species from the state of Morelos, Mexico, are described and illustrated, Malaxis ribana Espejo \& López-Ferrari and Habenaria uncata R. Jiménez, L. Sánchez \& García-Cruz. Both species were collected in the surroundings of the city of Cuernavaca.

Key words: Habenaria, Malaxis, Mexico, Morelos, Orchidaceae.

1 Dirección actual: Instituto de Ecología, A.C., Xalapa, Veracruz. 
Como resultado de la exploración intensiva llevada a cabo en el estado de Morelos durante los últimos seis años para la elaboración de la flora orquideológica del estado (Espejo et al., 2002), hemos descrito recientemente cuatro nuevos taxa (Espejo et al., 1998; García-Cruz et al., 2000). De igual manera, la revisión de material de herbario ha sido fuente importante para el descubrimiento de taxa nuevos (Salazar, 1997) y es el caso de las dos especies que aquí proponemos:

Malaxis ribana Espejo \& López Ferrari, sp. nov. (Fig. 1).

Herba perennis. Pseudobulbus hypogeus. Folia dua, anguste elliptica vel elliptica. Inflorescentia racemosa, racemo denso. Flores 42-150 in numero, non resupinati. Labellum triangulare, acuminatum, obconicum vel obpyramidale, concavum.

Hierba perenne, terrestre, de $24-42 \mathrm{~cm}$ de alto. Raíces escasas, originadas en la base del seudobulbo, de ca. $1 \mathrm{~mm}$ de diámetro, pilosas, blanquecinas. Seudobulbo hipógeo, ovoide a oblongo, de 2-3 cm de largo por 1.5-3.5 mm de ancho, cubierto por una vaina solitaria, tubular, estrecha, verdosa hacia el ápice, obtusa a aguda, de $5-13 \mathrm{~cm}$ de largo. Hojas dos, con una vaina tubular de $3-15 \mathrm{~cm}$ de largo, envolviendo la porción inferior del escapo; lámina de $6-11 \mathrm{~cm}$ de largo por 2-4.5 cm de ancho, angostamente elíptica a elíptica, en ocasiones largamente ovada, obtusa a redondeada, acuminada, la base decurrente, el margen entero. Inflorescencia erecta, originada de la parte apical del seudobulbo en desarrollo, racemosa, más larga que la hoja, con 42-150 flores; pedúnculo de 15-28 cm de largo por 1-1.5 mm de diámetro, racimo más o menos denso, sobre todo hacia la porción superior, de $8.5-20 \mathrm{~cm}$ de largo por 7-15 mm de diámetro. Brácteas florales ascendentes, verdes, triangulares, agudas, hialinas, de 1-2 mm de largo por ca. $1 \mathrm{~mm}$ de ancho. Flores ascendentes, de $5-6 \mathrm{~mm}$ de largo, amarillo-verdosas, no resupinadas. Ovario pedicelado subcilíndrico, acostillado, las costillas crespas, de 2-2.7 mm de largo por 0.7-0.8 mm de diámetro. Sépalo dorsal reflejo, ovado-lanceolado, obtuso, trinervado, de $3.6 \mathrm{~mm}$ de largo por $0.7 \mathrm{~mm}$ de ancho. Sépalos laterales erectos, oblicuos, oblongos, obtusos, trinervados, de $4 \mathrm{~mm}$ de largo por $1.3 \mathrm{~mm}$ de ancho. Pétalos recurvados, lineares, uninervados, de ca. $2 \mathrm{~mm}$ de largo por ca. $0.15 \mathrm{~mm}$ de ancho, el ápice redondeado. Labelo triangular cuando extendido, con los lados basales involutos, acuminado, abrazando a la columna en posición natural, de $3 \mathrm{~mm}$ de largo por $2 \mathrm{~mm}$ de ancho, con una quilla media longitudinal evidente, la base truncada, profundamente cóncavo hacia la parte basal, la concavidad triangular-ovada, de poco más de la mitad del largo del labelo. Columna corta, cuadrada, aplanada, truncada, de $0.6 \mathrm{~mm}$ de largo por $0.6 \mathrm{~mm}$ de ancho. Antera transversalmente oblonga, bilocular, de $0.2 \mathrm{~mm}$ de largo por $0.6 \mathrm{~mm}$ de ancho. Estigma ventral, semicircular, cóncavo. Cápsula elipsoide, de 6$7 \mathrm{~mm}$ de largo por $4 \mathrm{~mm}$ de diámetro.

Tipo: México, Morelos, municipio de Cuernavaca, E. Lyonnet 560800014 (Holotipo: MEXU 657289; Isotipo MEXU 657288).

Paratipos: México, Morelos, municipio de Cuernavaca, lomas de Tetela, VIVII.1953, E. Lyonnet 530600010 (MEXU 66335, MEXU 663338); municipio de Cuernavaca, Tetela del Monte, lomas, VIII.1955, E. Lyonnet 550800034 (MEXU 657282, MEXU 
Espejo et al.: Dos Especies Nuevas de Orquídeas del Estado de Morelos, México

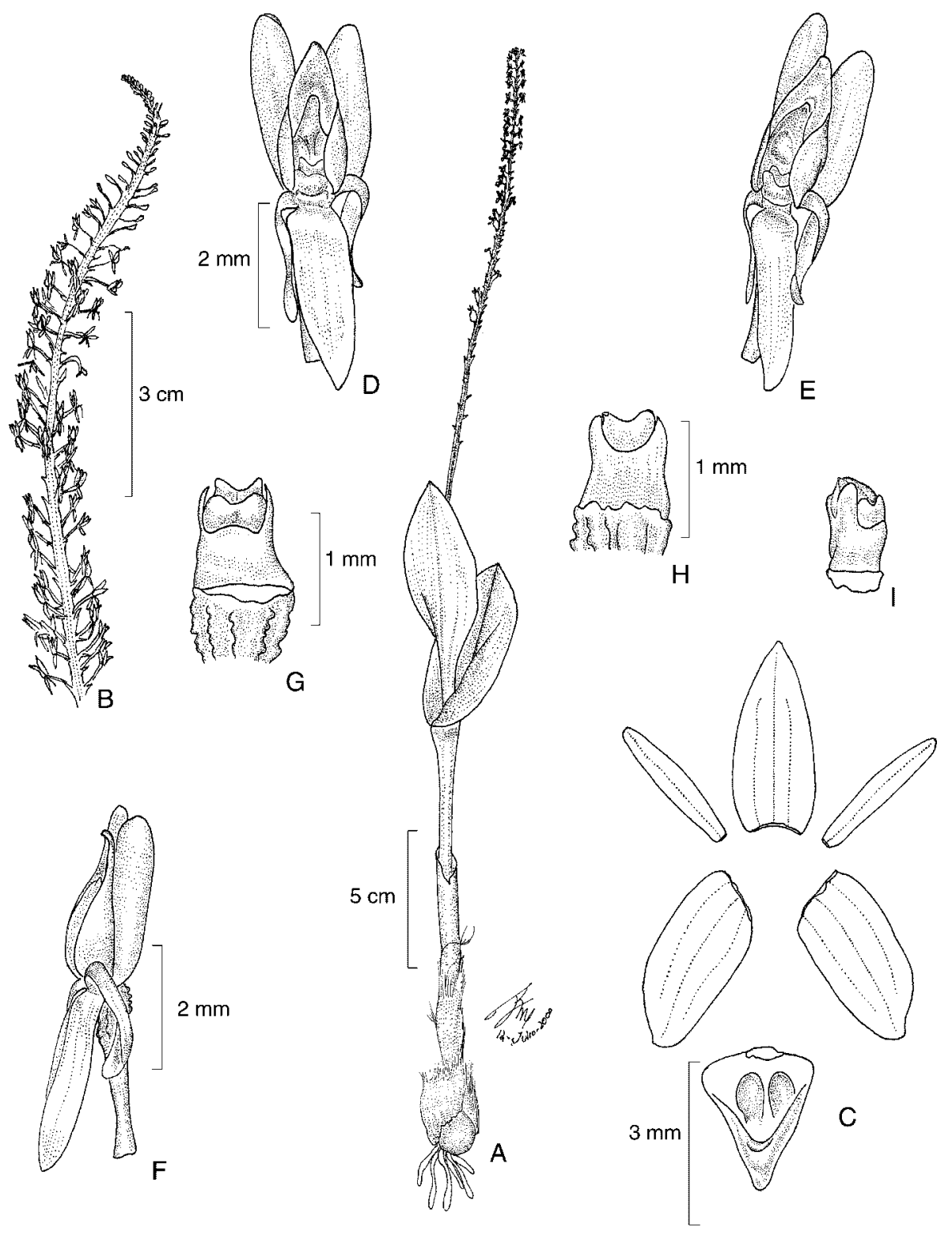

Fig. 1. Malaxis ribana Espejo \& López-Ferrari; A. Hábito de la planta; B. Detalle de la inflorescencia; C. Flor disecada; D. Flor, vista frontal; E. Flor vista de tres cuartos; F. Flor, vista lateral; G. Columna, vista dorsal; H. Columna, vista frontal; I. Columna, vista lateral. 
657283); municipio de Cuernavaca, north of Tetela in Barranca del Agua Zarca, in shade under Abies, 1600 m, H. D. Sawyer 982 (F 1260588).

Etimología: El nombre de la especie honra la memoria del Dr. Ramón Riba y Nava Esparza, maestro de numerosas generaciones de botánicos y excelente colega y amigo, desafortunadamente fallecido en diciembre de 2000.

Reconocimiento: Los pedicelos largos, el labelo triangular cuando extendido y profundamente cóncavo y abrazando a la columna en posición natural, permiten reconocer a la especie y distinguirla de Malaxis myurus (Lindl.) Kuntze, con la que puede confundirse en material herborizado (ver Cuadro 1).

Cuadro 1. Diferencias morfológicas entre Malaxis ribana y Malaxis myurus.

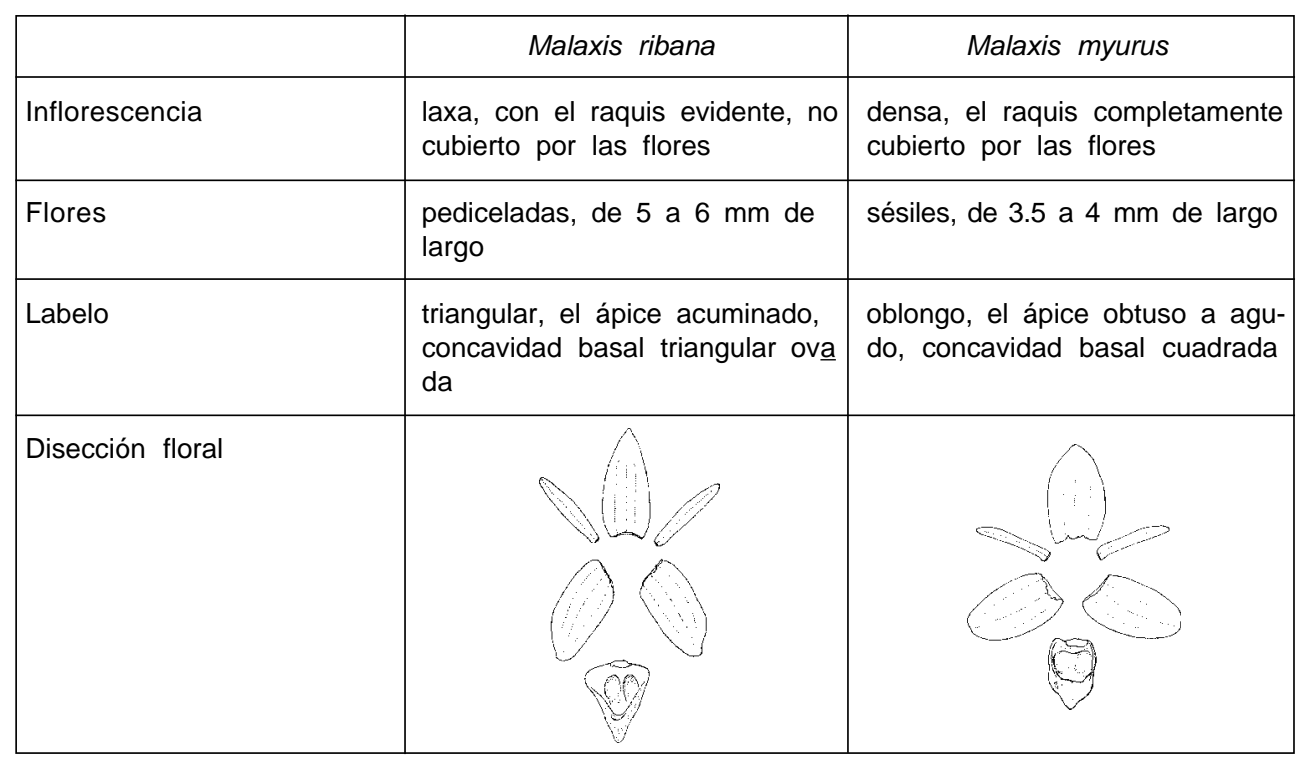

Malaxis ribana se conoce de diversas colecciones procedentes de los alrededores de Tetela del Monte, en el municipio de Cuernavaca. Infortunadamente esa parte del estado ha resentido en forma considerable los efectos de la perturbación antrópica y la especie no se ha vuelto a encontrar en tiempos recientes.

Habenaria uncata R. Jiménez, L. Sánchez \& García-Cruz, sp. nov. (Fig. 2).

Herba perennis, erecta, $35-40 \mathrm{~cm}$ alta. Folia 7-8, 3-8 cm longa, 1-3 cm lata, elliptica, margine integerrima. Inflorescentia racemosa. Flores $1.3-2 \mathrm{~cm}$ diametro, pallide virides. Calcar unciforme. 
Espejo et al.: Dos Especies Nuevas de Orquídeas del Estado de Morelos, México

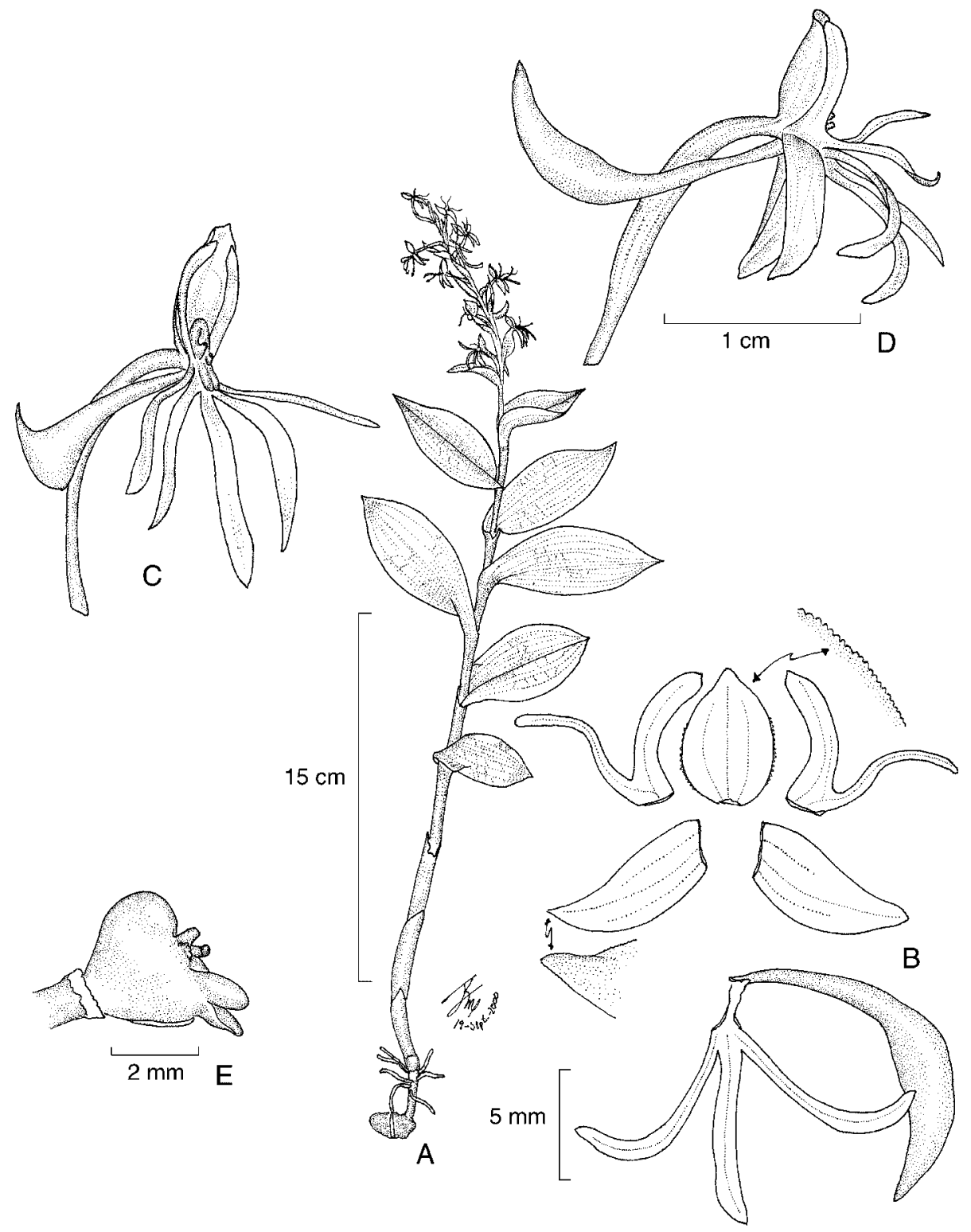

Fig. 2. Habenaria uncata R. Jiménez, L. Sánchez \& García-Cruz; A. Hábito de la planta; B. Flor disecada; C. Flor vista de tres cuartos; D. Flor, vista lateral; E. Columna, vista lateral. 
Hierba perenne, terrestre, erecta, de $35-40 \mathrm{~cm}$ de alto. Tuberoide ovoide a elipsoide, arrugado, de ca. $2 \mathrm{~cm}$ de largo por $1 \mathrm{~cm}$ de diámetro. Raíces delgadas, pubescentes. Hojas 7-8, elípticas, de $3-8 \mathrm{~cm}$ de largo por 1-3.5 cm de ancho, membranáceas, acuminadas, la base decurrente, el margen entero. Inflorescencia racemosa, de $6-9 \mathrm{~cm}$ de largo, el raquis algo comprimido y ligeramente aquillado, con 8-9 flores. Brácteas florales conduplicadas, lanceoladas, acuminadas, de 1-2 cm de largo por 5-18 $\mathrm{mm}$ de ancho, trinervadas, casi tan largas como el ovario o sobrepasándolo. Flores erectas a extendidas, de $1.3-2 \mathrm{~cm}$ de diámetro, simultáneas, verdosas. Ovario pedicelado, erecto, arqueado, rollizo, de 1.5-1.8 cm de largo. Sépalo dorsal erecto, cóncavo, ovado, agudo, de ca. $6 \mathrm{~mm}$ de largo por ca. $5 \mathrm{~mm}$ de ancho, trinervado, no carinado, diminutamente papiloso en el margen. Sépalos laterales reflexos, extendidos, oblicuamente triangular-lanceolados, agudos, mucronados, de ca. $7 \mathrm{~mm}$ de largo por $3 \mathrm{~mm}$ de ancho, trinervados, con una quilla diminutamente papilosa y baja en el dorso. Pétalos extendidos, bilobados, el lóbulo posterior en la misma posición que el sépalo dorsal y adherido a éste, arqueado, erecto, falcado, oblongo, agudo, de ca. $5 \mathrm{~mm}$ de largo por ca. $1 \mathrm{~mm}$ de ancho, uninervado; el lóbulo anterior filiforme, redondeado en el ápice, de ca. $9 \mathrm{~mm}$ de largo por ca. $0.6 \mathrm{~mm}$ de ancho, uninervado. Labelo trilobado, unguiculado, la uña subcuadrada, de ca. $1 \mathrm{~mm}$ de largo por ca. $1 \mathrm{~mm}$ de ancho; el lóbulo medio linearoblongo, agudo, de ca. $8 \mathrm{~mm}$ de largo por ca. $1 \mathrm{~mm}$ de ancho, uninervado; los lóbulos laterales divergentes en posición natural, lineares, arqueados en la porción distal, redondeados en el ápice, de ca. $9 \mathrm{~mm}$ de largo por ca. $0.9 \mathrm{~mm}$ de ancho, uninervados, sobrepasando apenas al lóbulo medio. Nectario dirigido hacia abajo, arqueado hacia atrás, recordando un anzuelo, claviforme, dilatado y aplanado en la porción apical, de ca. $1.5 \mathrm{~cm}$ de largo por $2.5 \mathrm{~mm}$ de diámetro, más largo que el lóbulo medio del labelo y un poco más largo que el ovario, el ápice agudo. Columna de ca. $3 \mathrm{~mm}$ de largo por ca. $2 \mathrm{~mm}$ de ancho; procesos estigmáticos con la superficie cóncava, formando un ángulo de $90^{\circ}$ con respecto a los canales de la antera; rostelo carnoso. Antera bilocular, los lóculos divergentes, de $1.6 \mathrm{~mm}$ de largo; los canales cortos, divergentes y ascendentes. Cápsula no vista.

Tipo: México, Morelos, municipio de Cuernavaca, lomas de Atzingo, VIII.1956, E. Lyonnet 560800010 (Holotipo: MEXU 663454; Isotipo MEXU 663455).

Etimología: El epíteto específico hace referencia a la forma de anzuelo o gancho que presenta el nectario.

Reconocimiento: La especie aquí propuesta es similar a Habenaria agapitae R. González \& Reynoso, pero difiere de ésta en los nectarios en forma de gancho y en las hojas menos numerosas, mucho más pequeñas, elípticas y con los márgenes enteros (ver Cuadro 2).

H. uncata se conoce sólo de la colección tipo y hasta ahora y pese al intensivo y sistemático trabajo de recolección realizado en el estado en los últimos años por los autores, no ha sido posible localizar nuevamente la planta. 
Espejo et al.: Dos Especies Nuevas de Orquídeas del Estado de Morelos, México

Cuadro 1. Diferencias morfológicas entre Habenaria uncata y $\mathrm{H}$. agapitae.

\begin{tabular}{|l|l|l|}
\hline Nectario & \multicolumn{1}{|c|}{ Habenaria uncata } & \multicolumn{1}{c|}{ Habenaria agapitae } \\
\hline Hojas & $\begin{array}{l}\text { de } 1.5 \mathrm{~cm} \times 2.5 \mathrm{~mm} \text {, dirigido ha } \\
\text { ción distal dilatada y aplanada }\end{array}$ & $\begin{array}{l}\text { de 1.8-2.5 cm x 1-2 mm, dirigido } \\
\text { hacia atrás, ligeramente arquea- } \\
\text { do, con la porción distal fusifor- } \\
\text { me, atenuada hacia el ápice }\end{array}$ \\
\hline Sépalo dorsal & $\begin{array}{l}7 \mathrm{u} 8, \text { elípticas, de 3-8 cm x } \\
1-3.5 \mathrm{~cm}, \text { margen entero }\end{array}$ & $\begin{array}{l}11 \text { a 19, obovadas a oblongo-róm } \\
\text { bicas o espatuladas, de 13-20 cm } \\
\text { x 3.5-4.7 cm, margen ondulado } \\
\text { a casi crespo }\end{array}$ \\
\hline Disección floral & $\begin{array}{l}\text { no carinado, con el ápice agudo } \\
\text { piloso margen diminutamente pa- }\end{array}$ & $\begin{array}{l}\text { carinado, con el ápice finamente } \\
\text { mucronado y el margen entero }\end{array}$ \\
\hline
\end{tabular}

\section{AGRADECIMIENTOS}

Agradecemos a Victoria Sosa la revisión crítica del manuscrito así como las sugerencias y comentarios al mismo. Roberto González Tamayo nos ayudó con sus observaciones acerca de la nueva especie de Habenaria. También deseamos expresar nuestra gratitud a Mario Sousa, curador del herbario MEXU por el préstamo de los ejemplares. Las figuras fueron realizadas por Rolando Jiménez Machorro.

\section{LITERATURA CITADA}

Espejo, A., A. R. López-Ferrari, J. García-Cruz, R. Jiménez Machorro \& L. Sánchez Saldaña. 1998. Dos nuevas adiciones a la orquideoflora mexicana. Sida 18(2): 411-418.

Espejo, A., J. García-Cruz, A. R. López-Ferrari, R. Jiménez Machorro \& L. Sánchez Saldaña. 2002. Orquídeas del Estado de Morelos. Orquídea (Méx.) 16: 1-392.

García Cruz, J., R. Jiménez Machorro, L. Sánchez Saldaña, A. Espejo \& A. R. López-Ferrari. 2000. Notas sobre el género Habenaria (Orchidaceae) en México. Acta Bot. Mex. 50: 27-38.

Salazar, G. 1997. A new species of Malaxis (Orchidaceae) from Morelos, Mexico. Brittonia 49: 449451.

Recibido en febrero de 2002. Aceptado en septiembre de 2002. 